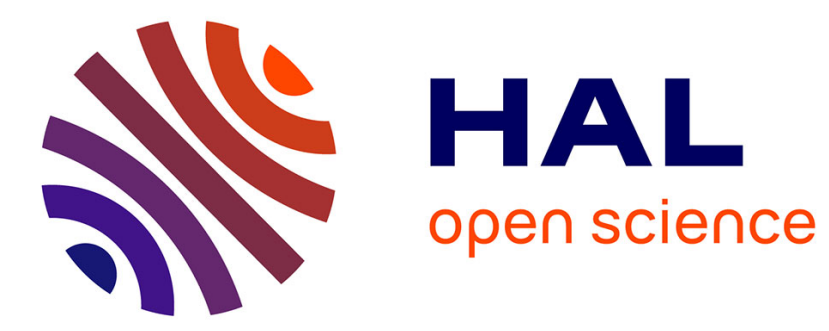

\title{
Techniques logiques de commande et animation de structures matricielles électroluminescentes
}

\author{
G. Batailler, J. Bernard, J.C. Besse, P. Garcia
}

\section{To cite this version:}

G. Batailler, J. Bernard, J.C. Besse, P. Garcia. Techniques logiques de commande et animation de structures matricielles électroluminescentes. Revue de Physique Appliquée, 1973, 8 (1), pp.75-81. 10.1051/rphysap:019730080107500 . jpa-00243656

\section{HAL Id: jpa-00243656 https://hal.science/jpa-00243656}

Submitted on 1 Jan 1973

HAL is a multi-disciplinary open access archive for the deposit and dissemination of scientific research documents, whether they are published or not. The documents may come from teaching and research institutions in France or abroad, or from public or private research centers.
L'archive ouverte pluridisciplinaire HAL, est destinée au dépôt et à la diffusion de documents scientifiques de niveau recherche, publiés ou non, émanant des établissements d'enseignement et de recherche français ou étrangers, des laboratoires publics ou privés. 
Classification

Physics abstracts

$17.28-18.40$

\title{
TECHNIQUES LOGIQUES DE COMMANDE ET ANIMATION DE STRUCTURES MATRICIELLES ÉLECTROLUMINESCENTES
}

\author{
G. BATAILLER, J. BERNARD, J. C. BESSE et P. GARCIA \\ Laboratoire de Luminescence 1, Faculté des Sciences \\ 40, avenue du Recteur-Pineau, 86000 Poitiers
}

(Reçu le 7 juillet 1972, revisé le 9 octobre 1972)

\begin{abstract}
Résumé. - Cet article traite de différentes techniques originales d'affichage d'informations statiques ou évolutives sur visualisateurs matriciels. Les supports utilisés sont des structures électroluminescentes au $\mathrm{ZnS}(\mathrm{Cu} / \mathrm{Cl}$ ) (à effet Destriau) ou à diodes à injection de porteurs au GaAsP. Des réalisations concrètes d'afficheurs ont permis d'évaluer les performances émissives de ces supports en fonction des différentes techniques exposées.
\end{abstract}

\begin{abstract}
This article presents some original techniques for visualisation of static or dynamic information on matrix displays. The used supports are electroluminescent devices composed of $\mathrm{ZnS}(\mathrm{Cu} / \mathrm{Cl})$ (Destriau effect) or of light emitting diodes (GaAsP). Prototypes showing emitting performances of this supports according to the treatment of information have been made.
\end{abstract}

Les matériaux électroluminescents qui se prêtent aisément à l'élaboration de structures matricielles $\mathrm{XY}$ de faibles dimensions et de bonne définition, constituent d'intéressants supports d'information pour les dispositifs actuels d'affichage.

En effet, l'excitation d'un point sur de tels écrans, résulte simplement de l'application d'une tension convenable (alternative pour les afficheurs à effet Destriau, continue pour ceux à injection de porteurs) entre un conducteur ligne et un conducteur colonne par l'intermédiaire d'interfaces ; quant à la transcrip-
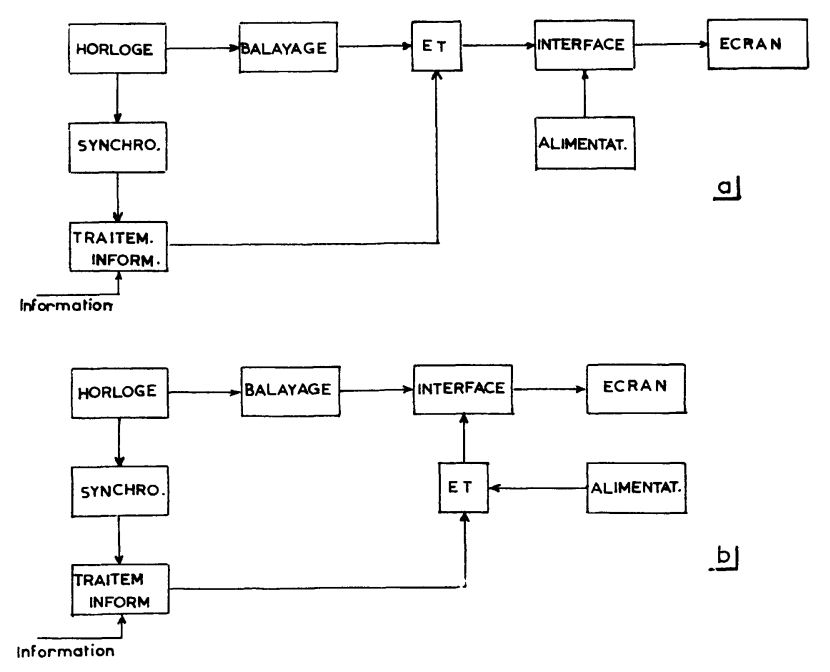

Fig. 1. - Principe de la visualisation d'une information sur écran matriciel; les signaux de balayage $(a)$ ou l'alimentation $(b)$ sont modulés par l'information incidente. tion d'un message, elle dépend d'ordres de sélection issus de l'information incidente, ordres agissant soit au niveau de la commande, soit au niveau de l'alimentation par l'intermédiaire de portes logiques (ET) (Fig. 1).

La manière dont se fait le traitement de l'information, et par conséquent l'adressage, permet de différencier les divers principes d'affichage; les modes de balayage de l'écran se définissent à partir de l'ordre et de la vitesse de scrutation de tous les points.

Dans ce qui suit, sont présentés divers traitements originaux de l'information, mémorisée ou évolutive, ainsi que leur influence sur les caractéristiques d'émission de tels afficheurs, notamment le contraste pour ceux à effet Destriau.

1. Techniques d'affichage. - Bien qu'il ne s'agisse ici que d'affichage dynamique [1], deux types d'informations incidentes peuvent être considérés : l'information mémorisée qui n'évolue pas pendant au moins un cycle complet de balayage et l'information évolutive qui se modifie continuellement dans le temps.

1.1 AfFichage D'INFORMATIONS MÉMORISÉEs. D'une façon générale, le balayage simultané par exemple de type télévision [2] d'une matrice électroluminescente et d'une mémoire morte de même configuration, permet un affichage répétitif des informations contenues dans cette mémoire. Un point de l'écran ne s'illumine que lorsqu'il est adressé par le balayage et qu'un ordre simultané délivré par la mémoire n'inhibe pas cet adressage (principe Fig. 1a). 
Ce principe d'affichage d'informations par modulation de signaux de balayage est communément appliqué dans les afficheurs alphanumériques classiques utilisant les mémoires mortes intégrées [3]. Dans ce cas, la représentation de tous les caractères usuels conduit à des mémoires d'environ 2 kbits.

Cependant leur dimension peut être considérablement réduite par un traitement différent de l'information :

- la définition des caractères étant généralement de $5 \times 7$ points, il est intéressant de faire correspondre la géométrie de tout caractère à visualiser avec le profil de 7 tensions génératrices de séquences (Fig. 2) [4], [5] ;

- l'ensemble des symboles alphanumériques habituels peut en effet, être élaboré à partir de seulement 16 séquences différentes, ce qui conduit à une mémoire de 80 bits.
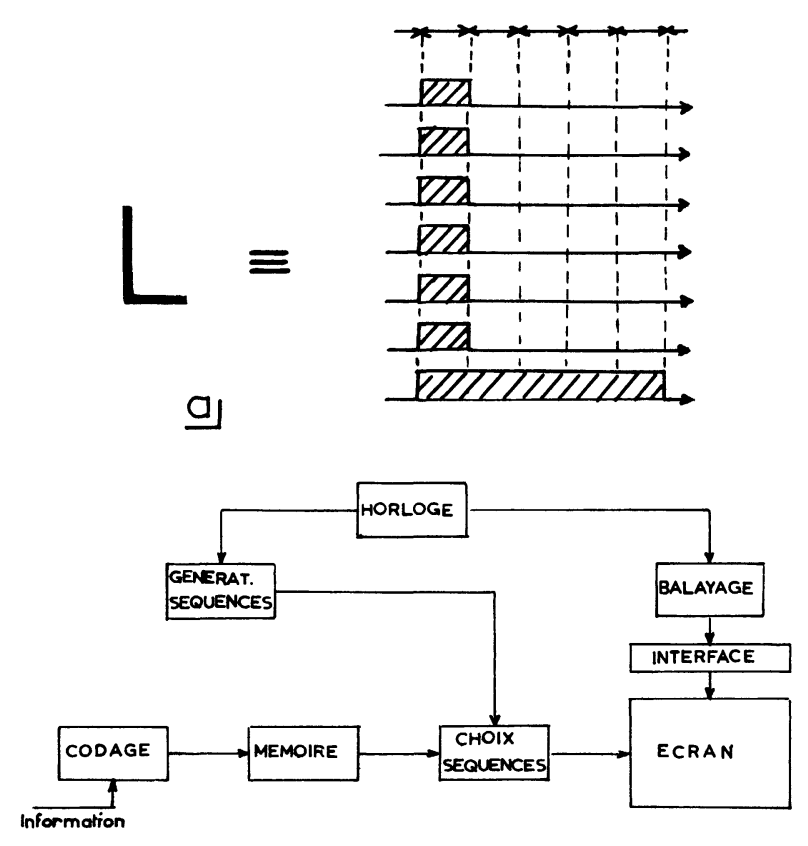

b)

FIG. 2. - Affichage d'informations par génération de séquences. a) Image séquentielle d'une lettre ; $b$ ) Principe de la visualisation : le codage binaire de chaque caractère permet le choix répétitif de ses 7 séquences images et leur affichage

L'excitation des points représentatifs du caractère peut alors se faire par la coïncidence de l'adressage colonne et du niveau haut des séquences convenablement choisies le balayage étant simultané sur les 7 lignes. Dans ce cas, la mémorisation préalable d'un message dans un registre à décalage bouclé permet de le visualiser en permanence par répétition.

1.2 Affichage DE Signaux Évolutifs. - Quelle que soit la technique utilisée, la position d'un point sur une colonne de l'écran représente l'amplitude du signal incident à un instant donné.

Si le balayage reste de type télévision colonne par colonne, l'adressage d'un point résulte d'une transformation amplitude-temps-espace [1], [6], [7].
En effet, en comparant les amplitudes du signal d'entrée et d'une tension de référence en marches d'escalier synchrone avec le balayage, sortie d'un convertisseur digital-analogique, dont la largeur des marches est égale au pas de ce balayage, l'instant de leur égalité unique traduit une position sur une colonne de l'écran. L'illumination du point représentatif résulte de l'application de la tension d'alimentation à partir de cet instant et pendant une durée au plus égale à un pas de balayage (Fig. 3).

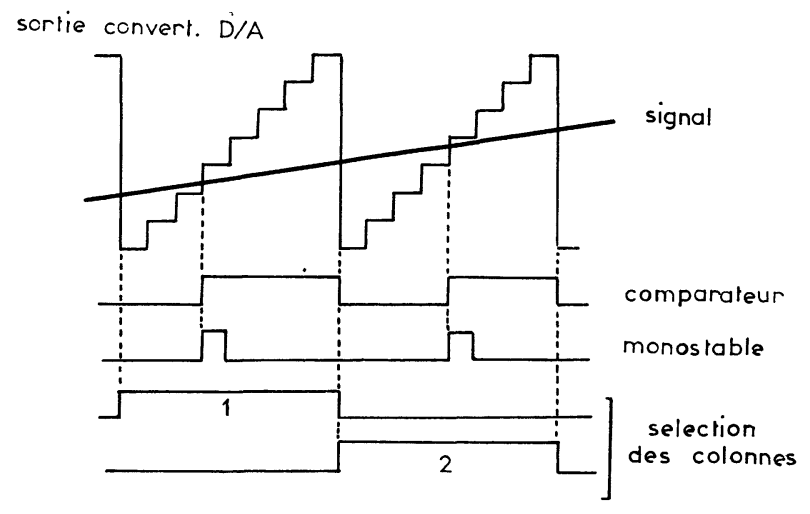

Fig. 3. - Principe de l'affichage de signaux évolutifs.

Ce principe conduit nécessairement à une luminosité relativement faible de l'image représentée, étant donné la courte durée d'excitation du point (un pas de balayage) et le temps important séparant deux illuminations sur deux colonnes adjacentes ( $n$ pas de balayage si $n$ est l'ordre de la matrice).

Pour pallier cet inconvénient, une codification de la valeur de l'amplitude du signal incident lors de l'égalité est nécessaire.

Cette conversion analogique-numérique (binaire) permet en effet une semi-mémorisation par l'intermédiaire de deux mémoires logiques disposées en cascade, l'une étant commandée lors de l'égalité, l'autre à chaque début du cycle de conversion. La seconde mémoire attaquant directement l'interface, l'illumination du point représentant l'amplitude du signal incident à l'instant considéré, se produit pendant toute la durée du balayage de la colonne suivante (Fig. 4) [8], [9].

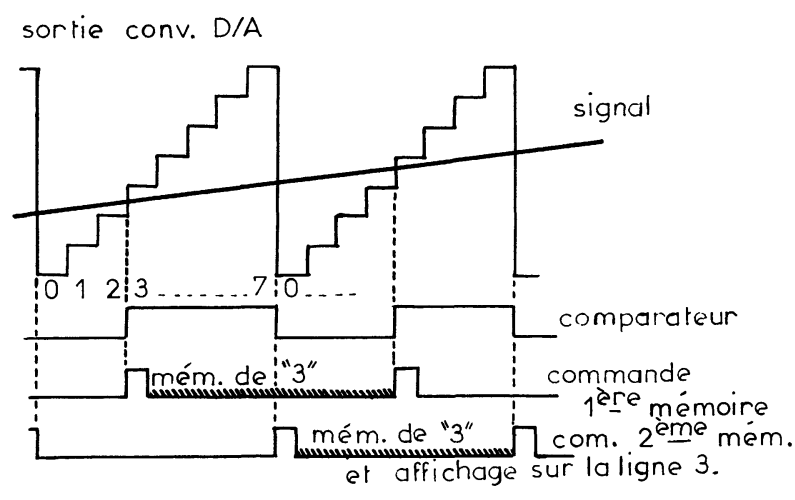

Fig. 4. - Affichage de signaux évolutifs par semi-mémorisation. 
Ainsi tous les points représentatifs's seront excités pendant un temps égal (valant $n$ pas de balayage, si $n$ est le nombre de lignes de la matrice) et à tout instant au moins un point est excité.

Dans ce second cas, un fonctionnement par commande "XY» peut être envisagé. Le compteur de scrutation par adjacence des colonnes est remplacé par un dispositif de traitement de l'information identique à celui des lignes et synchrone avec ce dernier. Les coordonnées $x$ et $y$ d'un point codifiées et mémorisées lors d'un cycle de conversion sont affichées lors du cycle suivant et définissent un point unique.

Un appareil réalisant cette fonction, commandé par une horloge de fréquence $2 \mathrm{MHz}$ permet la transcription de signaux de fréquence pouvant avoisiner $8 \mathrm{kHz}$ (fonctionnement en balayage ou «X, Y» (Fig. 5)).

Quant aux résultats que l'on peut escompter à l'aide d'un tel dispositif, ils sont représentés sur la figure 6 , pour un fonctionnement en balayage.

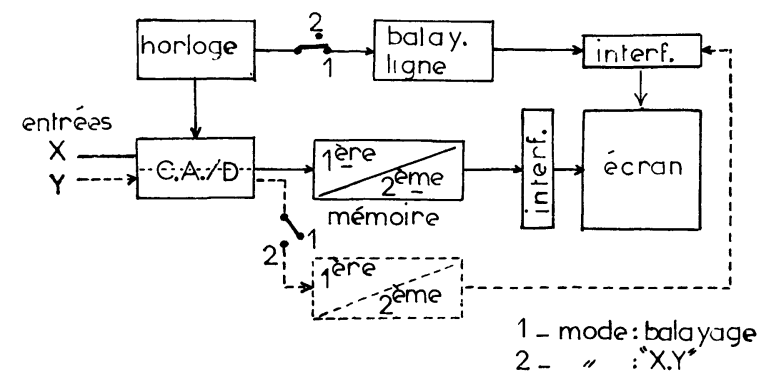

FIG. 5. - Schéma de principe d'un « oscilloscope matriciel ».
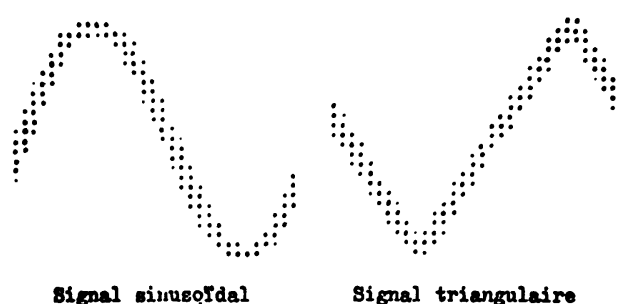

Frsquence $=8 \mathrm{~Hz}$.

Signal triangulaire

Fréquence $=8 \mathrm{~Hz}$.

FIG. 6. - Photographies de signaux visualisés sur « oscilloscope matriciel $»(31 \times 31$ points $)$

2. Influence du milieu de formation de l'image. - Les techniques précédemment envisagées sont applicables à tous les types de matrices à adressage lignes-colonnes. Seuls l'alimentation et par là même l'interface doivent être modifiés. Par exemple, l'écran matriciel au $\mathrm{ZnS}(\mathrm{Cu} / \mathrm{Cl})$ nécessite une tension d'excitation alternative de l'ordre de $150 \mathrm{~V}$, ce qui implique un interface à composants haute tension (thyristors, triacs, transistors HT...) tandis qu'un visualisateur à diodes électroluminescentes au GaAsP est excité par une tension continue de faible niveau $(2 \mathrm{~V}, 20 \mathrm{~mA})$, n'imposant pas d'interface particulier.

S'il n'existe donc pas de différences essentielles pour les techniques d'affichage, les résultats au niveau de l'écran dépendront surtout du matériau constituant celui-ci. Son caractère actif ou passif, sa rapidité de réponse, sa luminance, le contraste existant entre l'illumination de deux points excités différemment, seront les diverses particularités qui détermineront le choix d'un visualisateur.

2.1 Cas D'une matrice au $\mathrm{ZnS}(\mathrm{Cu} / \mathrm{Cl})$ [2]. L'excitation alternative d'un élément d'une telle matrice fait apparaitre en plus de l'illumination de celui-ci (environ 5 FL pour une tension d'excitation de $150 \mathrm{~V}$ ), une illumination partielle des éléments de la ligne et de la colonne excitées. Cette illumination est due à la présence de tensions parasites, avoisinant $V / 2$ si l'ordre $n$ de la matrice supposée carrée est assez grand, qui proviennent de la distribution de la tension $V$ d'excitation à travers cette structure de caractère passif (Fig. 7) [10], [11].

En effet, en considérant l'impédance $Z$ d'une cellule élémentaire et des conducteurs idéaux, il est aisé de calculer à partir du schéma simplifié de l'écran matri-

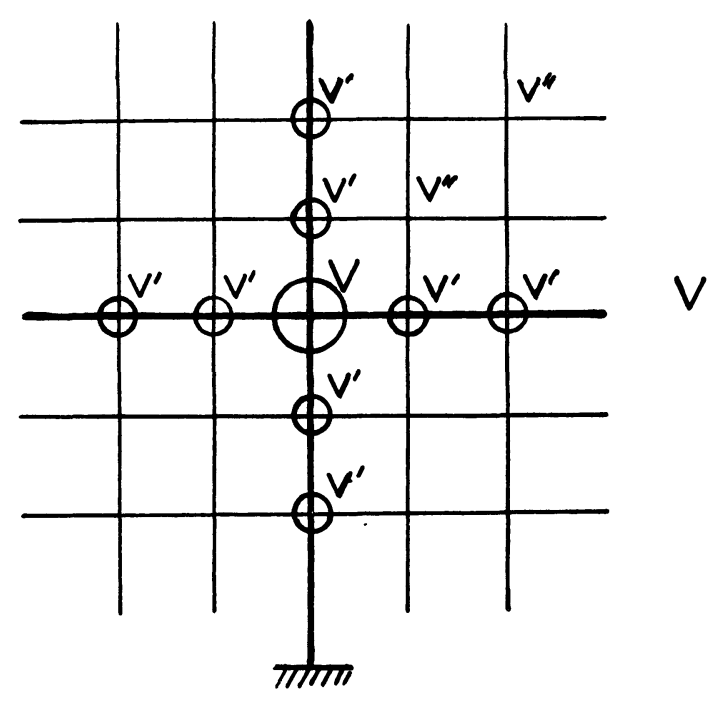

a

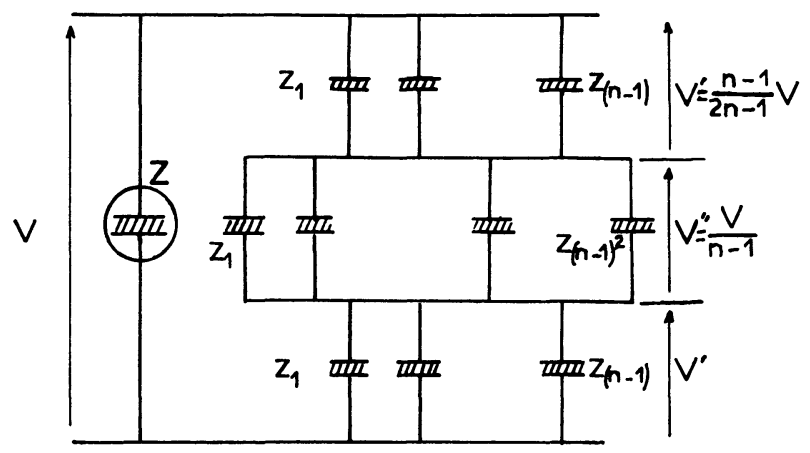

b)

Fig. 7. - Répartition schématique des tensions alternatives sur une matrice à effet Destriau. a) Mise en évidence d'une croix parasite; $b$ ) Détermination des tensions parasites à partir du schéma électrique comportant des conducteurs idéaux. 
ciel, la tension existant aux bornes des différents points de la «croix », tension dont la valeur est :

$$
V^{\prime}=\frac{n-1}{2 n-1} V
$$

lorsqu'un seul croisement est excité.

En raison de la loi luminance-tension du matériau

$$
L=A \exp \left(-\frac{B}{\sqrt{V}}\right)
$$

le contraste théorique entre le point excité et ceux de la croix parasite (rapport des luminances intégrées pour les deux tensions $V$ et $V / 2$ ) peut s'exprimer par :

$$
C=\exp \left(\frac{0,414 B}{\sqrt{V}}\right)
$$

$B$ étant pratiquement constant et fonction du matériau employé, $C$ est d'autant meilleur que la tension d'excitation est faible, mais à une tension peu élevée correspond une luminance insuffisante, et un compromis s'impose.

En fait, pour les conditions normales d'utilisation des écrans étudiés, le contraste que l'on peut espérer est de 1 à 20 , si l'on fixe la fréquence d'excitation à une valeur supérieure à la fréquence correspondant au coude de la courbe luminance-fréquence.

Ce contraste, relativement faible pour l'œil en statique, ajouté à la faible luminance de ce type d'écran, ne permet pas d'escompter une bonne visualisation lors d'un fonctionnement dynamique puisqu'un fond lumineux important va exister.
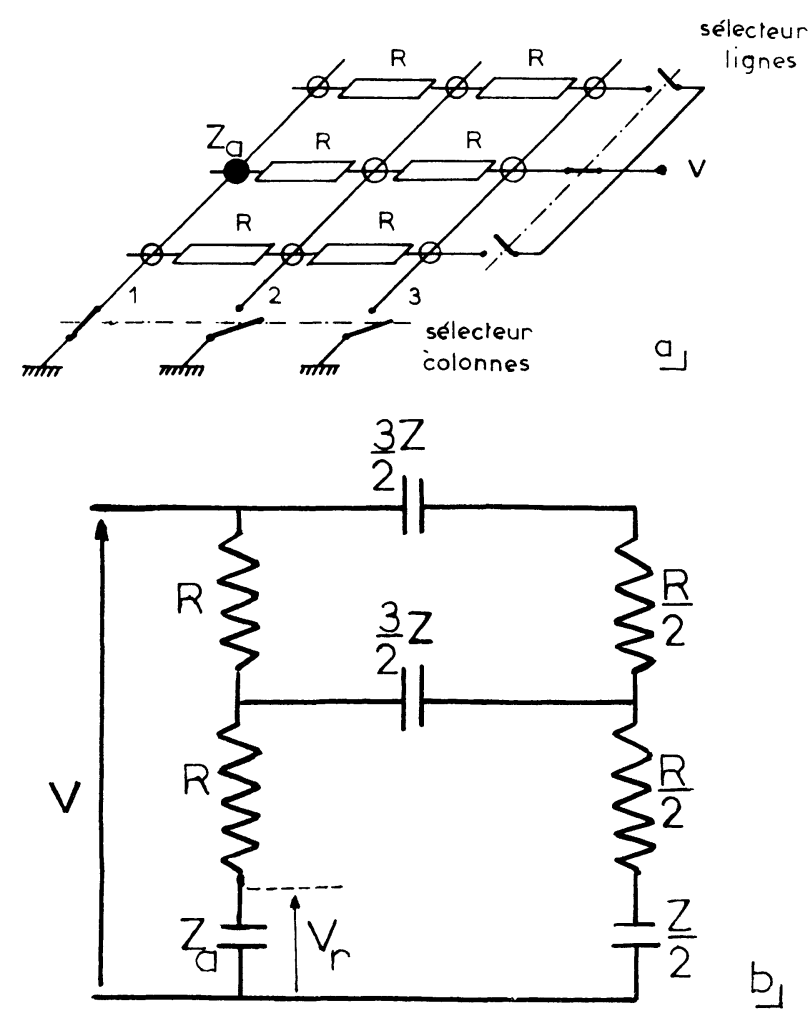

FIG. 8. - a) Introduction des résistances linéiques des conducteurs sur le schéma simplifié d'une matrice $3 \times 3$ points; b) Exemple de schéma électrique permettant le calcul des tensions aux différents points de l'écran.
De plus, ce fond lumineux n'est pas uniforme. En effet, lorsque le point excité se déplace sur la matrice, son intensité lumineuse relevée à l'aide d'un photomultiplicateur, ainsi que celle de la croix parasite, est différente suivant sa position.

Ce résultat provient principalement de la constitution de l'écran et surtout de la résistance linéique des conducteurs transparents en $\mathrm{SnO}_{2}$ (Fig. 8).

En introduisant la résistance linéique $R$, le schéma équivalent d'une matrice de $3 \times 3$ points devient celui de la figure $8 b$ et il est alors possible en utilisant la méthode des graphes de transfert de calculer la valeur des tensions $V_{\mathrm{r}}$ en chaque point à partir de la tension d'excitation $V$ et de valeurs approximatives pour l'impédance $Z$ et la résistance $R$.

Un exemple de la répartition des tensions $V^{\prime}$, $V^{\prime \prime}, V_{\mathrm{r}}=f(V)$ et du rapport de tension $V^{\prime}, V^{\prime \prime}=f\left(V_{\mathrm{r}}\right)$ affectant directement le contraste en fonction de la position du point excité est donné sur la figure 9.
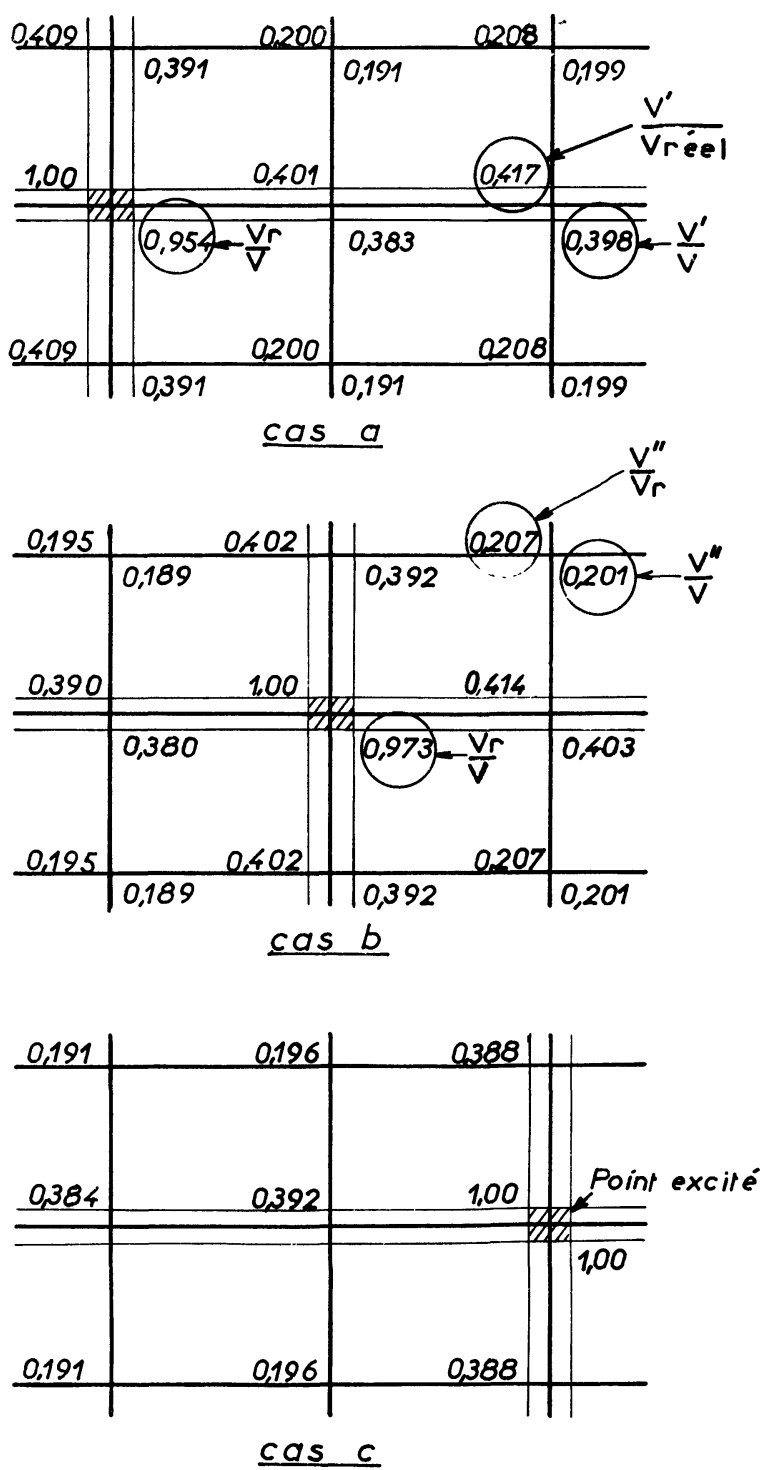

FIG. 9. - Répartition des tensions sur une matrice $3 \times 3$ en fonction de la position du point excité sur chacune des trois colonnes $(a, b, c)$ 


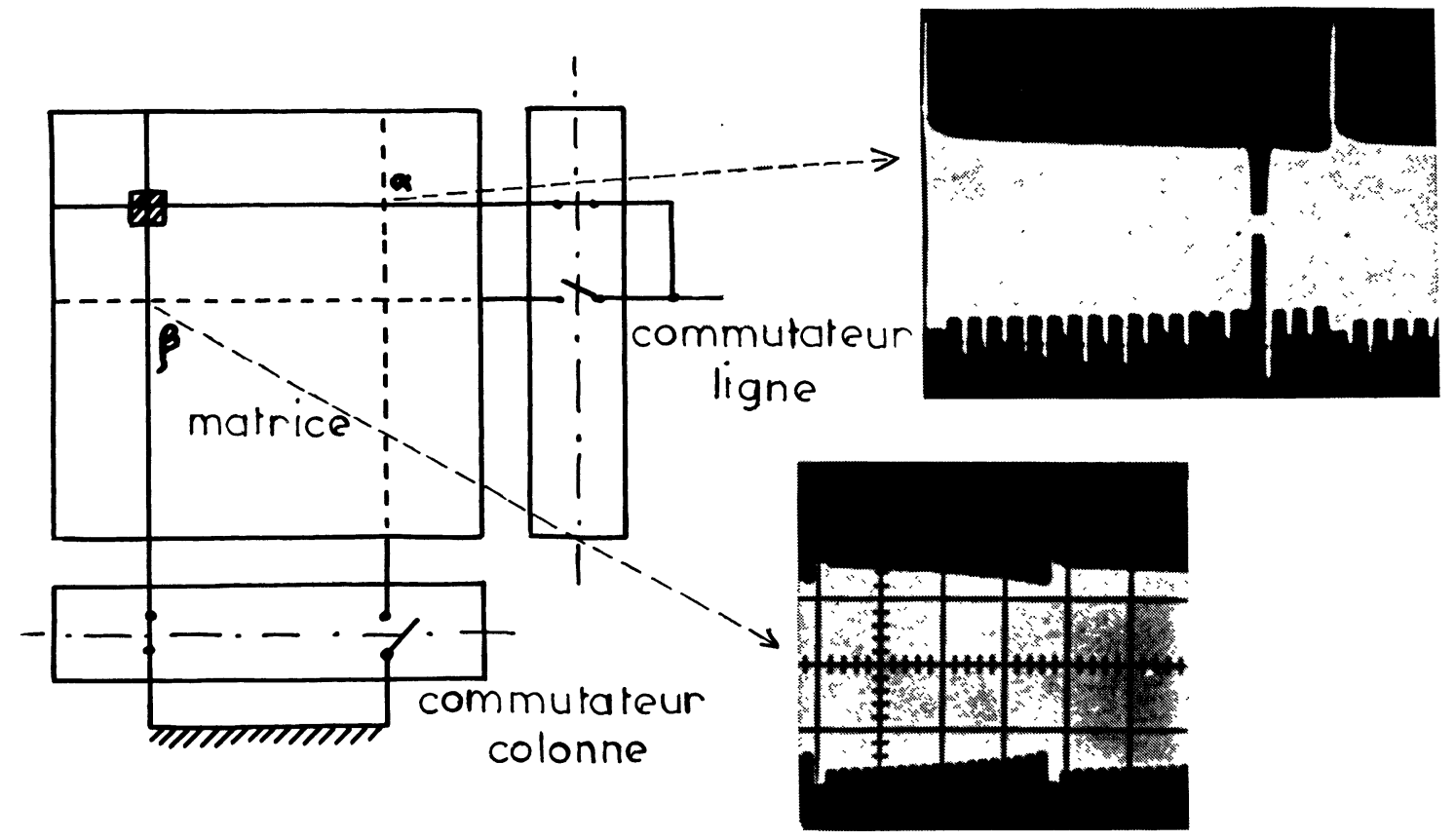

FIG. 10. - Evolution de la tension en $\alpha$ et $\beta$ lorsque le point excité se déplace de la gauche vers la droite sur la matrice.

Ces résultats corroborent parfaitement les mesures qui peuvent être faites sur les tensions, mesures qui sont reportées à la figure 10 , et la variation de luminance d'un point lors du balayage (Fig. 11).

(a)

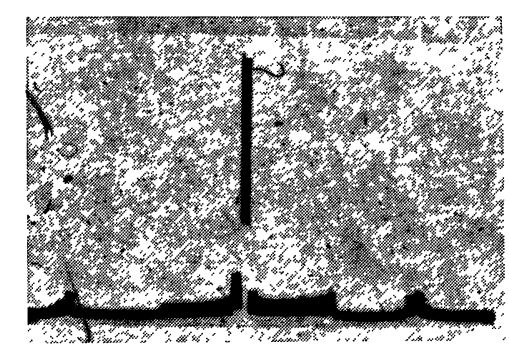

(b)

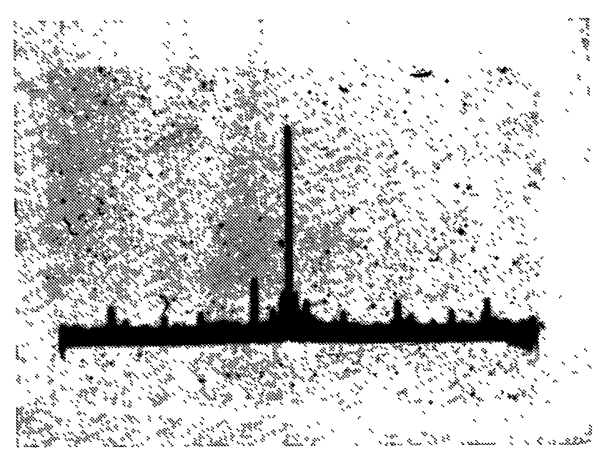

FIG. 11. - Evolution de la luminance d'un point lors du balayage d'une ligne : a) Balayage lent $(25 \mathrm{~Hz})$; contraste voisin de 1 à $20 ; b)$ Balayage rapide $(5 \mathrm{kHz})$.

En effet, pour le balayage rapide $(5 \mathrm{kHz})$ nécessaire à la visualisation sur l'écran considéré, le fond lumineux continu est plus important que pour un balayage lent et il s'y ajoute de plus des pics de luminance indésirables, pics dus à la discontinuité des tensions lors de chaque commutation. Cette discontinuité provient de l'effet capacitif des cellules élémentaires, et elle est plus grande si l'alimentation est polarisée en continu. La figure 12 explique sommairement cette discontinuité de même qu'elle montre la composition de la tension parasite sur une ligne relevée à l'oscilloscope.

D'autre part, d'autres inconvénients, inhérents au matériau, sont à considérer :

- pour un balayage rapide, l'excitation d'un point devient quasi impulsionnelle et du fait que le temps de réponse à la mise sous tension d'une cellule élémentaire est relativement importante et fonction de la fréquence d'excitation (10 périodes excitatrices pour une fréquence de $10 \mathrm{kHz}$ ) la luminance tend à diminuer. Ceci conduit à choisir la fréquence d'excitation en fonction de celle du balayage ou à faire appel à une semi-mémorisation de l'information ;

- le vieillissement irréversible de la poudre électroluminescente, qui conduit à un affaiblissement quasi exponentiel de la luminance en fonction de la durée de fonctionnement. Ce phénomène, surtout sensible dans les cellules de type organique, conduit également à une modification du schéma électrique équivalent et peut donc avoir des incidences sur le contraste [8], [12].

Remarque. - Il est à noter que lorsqu'il s'agit d'un balayage par faisceaux (par exemple 7 lignes balayées simultanément colonne par colonne) la tension parasite prend une valeur différente sur les colonnes et sur les lignes.

A chaque pas de balayage, la tension parasite colonne vaut :

$$
\frac{n^{\prime}(n-1)}{n^{\prime}(n-1)+n} V
$$




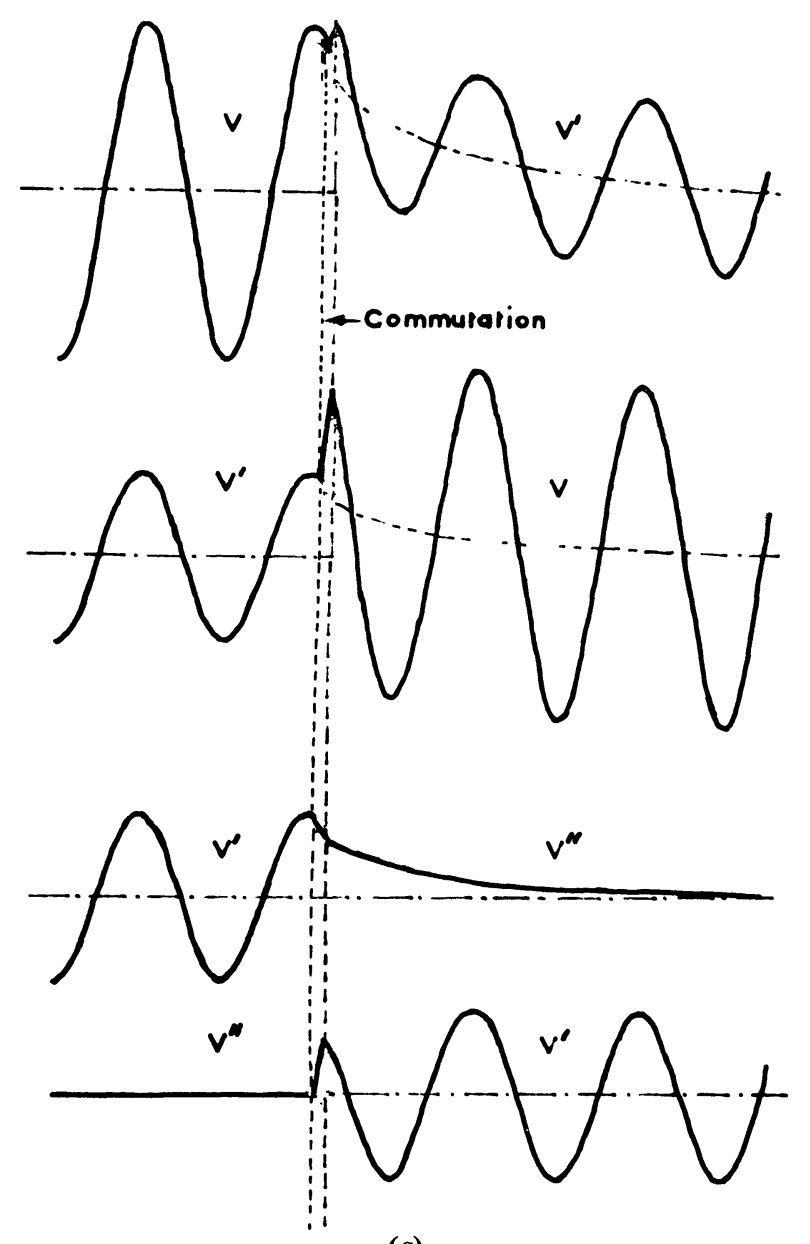

(a)

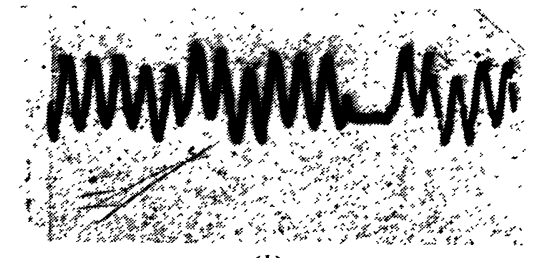

(b)

FIG. 12. - Discontinuité des tensions : $a$ ) Evolution qualitative des tensions lors d'une commutation; $b$ ) Relevé expérimental lors du balayage, de la tension parasite $\left(V^{\prime}\right)$ à partir des conducteurs définissant ce point.

tandis que la tension parasite lignes vaut :

$$
\frac{n-n^{\prime}}{n^{\prime}(n-1)+n} V
$$

si $n^{\prime}$ est le nombre de lignes excitées simultanément, ceci conduisant à une non-uniformité plus importante et un contraste moins bon.
2.2 Cas D'un éCran a Diodes au GaAsP. - Un des avantages des diodes électroluminescentes en structure matricielle sur les écrans à effet Destriau est l'obtention d'une image présentant un contraste infini. En effet, sur de tels afficheurs, en raison du caractère unidirectionnel de ces éléments, seule la diode sélectionnée est excitée. Sur toutes les autres branches du réseau une diode polarisée en inverse ne laisse passer qu'un courant de fuite trop faible pour commander éventuellement d'autres diodes.

Ces diodes présentent une luminance relativement élevée ( $750 \mathrm{FL}$ sous $20 \mathrm{~mA}, \lambda=6500 \AA$ ), leur durée de vie est pratiquement infinie, et en raison de leur réponse extrêmement rapide (de l'ordre de la nanoseconde) et de leur bonne tenue aux forts courants impulsionnels, elles se prêtent facilement aux techniques de commandes envisagées.

3. Conclusion. - Les écrans matriciels à diodes électroluminescentes semblent a priori plus intéressants que ceux à effet Destriau pour l'affichage d'informations. En effet, la luminance importante, la bonne rapidité de réponse, la simplicité de l'électronique de commande, ainsi que l'excellent contraste obtenu, semblent être des avantages déterminants en faveur des afficheurs à injection de porteurs.

Cependant, la faible consommation, le coût peu élevé de l'écran, une définition d'image comparable font que les visualisateurs à électroluminescence intrinsèque ne doivent pas être totalement rejetés. De plus, le contraste peut être amélioré soit par l'application d'une tension en opposition sur les conducteurs non sélectionnés bien qu'au détriment d'une électronique plus lourde, soit par adjonction d'une couche à impédance variable; de même la durée de vie peut être notablement améliorée dans le cas des cellules métalcéramique $(3000 \mathrm{H}$ sous $200 \mathrm{~V}, 400 \mathrm{~Hz})$.

Quant aux techniques de traitement de l'information, elles bénéficient de l'importance prise par l'électronique digitale, ce qui se traduit par une baisse sensible du prix du visualisateur.

Les applications de tels dispositifs sont multiples et peuvent être destinées soit à la transcription visuelle plane d'informations de type quelconque (schémas, reconnaissance de forme ou informations alphanumériques dans les périphériques de calculateurs ou les appareils de mesure) soit à la visualisation d'informations vidéo (image télévision [13] ou plus simplement la visualisation oscilloscopique de signaux électriques).

\section{Bibliographie}

[1] Tran R., Besse J., Batailler G., « Sur une dynamique de mise sous tension d'une matrice de cellules électroluminescentes et l'interprétation visuelle de phénomènes fixes ou variables dans le temps. » C. R. Acad. Sci. Paris (1970).
[2] Besse J. C., "Contribution à l'étude d'un dispositif de visualisation électroluminescente en structure matricielle. » Thèse de Docteur-Ingénieur Poitiers, décembre 1971.

[3] Mostek Product Guide, janvier 1971. 
[4] Garcia P., Bernard J., « Affichage alphanumérique sur écran matriciel à diodes au GaAsP par génération de séquences. " Electronique et microélectronique industrielles, No 153 , mars 1972.

[5] Garcia P., "Application de la logique à l'affichage alphanumérique séquentiel sur écran matriciel électroluminescent au GaAsP. » Thèse de Docteur de $3^{\mathrm{e}}$ Cycle, Poitiers, juin 1971.

[6] Garcia P., "Affichage numérique sur un écran matriciel à diodes au GaAsP ». DEA, Poitiers, juin 1971.

[7] Tran R., «Contribution à l'étude d'un visualisateur matriciel électroluminescent de signaux évolutifs ». Thèse d'université, Poitiers, mars 1971.

[8] Bernard J., "Contribution à l'étude du vieillissement des visualisateurs électroluminescents au $\mathrm{ZnS} / \mathrm{Cu}$ et réalisation d'un "oscilloscope matriciel» à diodes au GaAsP à affichage semi-mémorisé ». Thèse de Docteur-Ingénieur, Poitiers, février 1972.
[9] BERnARD J., «Affichage d'informations dynamiques par semi-mémorisation sur écran électroluminescent. Réalisation d'un oscilloscope matriciel $»$. Electronique et microelectrique industrielle $\mathrm{n}^{\circ} 161$, octobre 1972.

[10] Deschamps, « Dispositifs optoélectroniques réalisés à la SAT ». Revue Télécom., avril 1969.

[11] YveY, ThORTon, « Preparation and properties of electroluminescent phosphors for display devices ». IRE transactions on electron devices, juillet 1961.

[12] Hahn, Mimkes, " Aging mechanism of $\mathrm{ZnS} / \mathrm{Cu}$ electroluminescence ». J. Phys. Chem. Solids 29 (1968) 1287.

[13] YoshiYama, «Lighting the way to flat-screen TV ». Electronics, 1969.

[14] Documentation technique. Monsanto, Hewlet Packard, Bell telephone, RCA. 